\title{
Smooth and sharp creation of a pointlike source for a $(3+1)$-dimensional quantum field
}

\author{
L. J. Zhou, ${ }^{1,2,3,4, *}$ Margaret E. Carrington, ${ }^{1,2, \dagger}$ Gabor Kunstatter, ${ }^{2,3}$ and Jorma Louko ${ }^{5, \S}$ \\ ${ }^{1}$ Department of Physics, Brandon University, Brandon, Manitoba, R7A 6A9 Canada \\ ${ }^{2}$ Winnipeg Institute for Theoretical Physics, Winnipeg, Manitoba \\ ${ }^{3}$ Department of Physics, University of Winnipeg, Winnipeg, Manitoba, R3B 2E9 Canada \\ ${ }^{4}$ Department of Physics and Astronomy, University of Manitoba, Winnipeg, Manitoba, R3T 2N2 Canada \\ ${ }^{5}$ School of Mathematical Sciences, University of Nottingham, Nottingham NG7 2RD, United Kingdom
}

(Received 6 January 2017; published 10 April 2017)

\begin{abstract}
We analyze the smooth and sharp creation of a pointlike source for a quantized massless scalar field in $(3+1)$-dimensional Minkowski spacetime, as a model for the breakdown of correlations that has been proposed to occur at the horizon of an evaporating black hole. The creation is implemented by a timedependent self-adjointness parameter at the excised spatial origin. In a smooth creation, the renormalized energy density $\left\langle T_{00}\right\rangle$ is well defined away from the source, but it is unbounded both above and below: the outgoing pulse contains an infinite negative energy, while a cloud of infinite positive energy lingers near the fully-formed source. In the sharp creation limit, $\left\langle T_{00}\right\rangle$ diverges everywhere in the timelike future of the creation event, and so does the response of an Unruh-DeWitt detector that operates in the timelike future of the creation event. The source creation is significantly more singular than the corresponding process in $1+1$ dimensions, analyzed previously, and it may be sufficiently singular to break quantum correlations as proposed in a black hole spacetime.
\end{abstract}

DOI: 10.1103/PhysRevD.95.085007

\section{INTRODUCTION}

In quantum field theory, it has been long known that a time dependent boundary condition or a time dependent metric can create particles and energy flows. Parker's pioneering work showed that a Klein-Gordon field on an expanding cosmological spacetime undergoes particle creation [1]. Moore showed that particle creation can be induced by varying the length of a cavity [2], while Candelas and Deutsch showed that even a single accelerating mirror can induce a flux of particles and energy [3]; this phenomenon is now known as the Dynamical (or nonstationary) Casimir Effect, and it was observed in 2011 using a photon analogue system [4]. The most celebrated example is Hawking's prediction of black hole radiation [5], whose observation in analogue quantum systems may be at the threshold of current technology [6,7].

In order to reconcile the thermal character of Hawking radiation with fundamental unitarity of quantum theory, it has been proposed [8-14] that the horizon of a radiating black hole could be more singular than the conventional picture of quantum fields on the classical black hole spacetime suggests [15-17]. While detailed modeling of this possible singularity remains elusive, the key proposed feature is that the singularity should break down

\footnotetext{
*zhoulingjunjeff@gmail.com

†carrington@brandonu.ca

*gkunstatter@uwinnipeg.ca

§orma.louko@nottingham.ac.uk
}

correlations between the two sides of the horizon. A context in which such breaking of correlations can be studied is quantum field theory on a fixed background spacetime. One way to do this is to write down by hand a quantum state in which the correlations are absent $[18,19]$. Another is to allow an impermeable wall to develop where initially there was none [20-23]. The purpose of the present paper is to improve the understanding of the latter scenario.

When the impermeable wall is inserted quickly, a surprising feature emerges: for a massless scalar field in $1+1$ dimensions, the energy transmitted into the field diverges in the limit of rapid wall creation, but the response of an Unruh-DeWitt detector $[24,25]$ crossing this pulse of diverging energy remains finite [22]. The finite detector response casts doubt on the ability of wall creation, however rapid, to break down quantum correlations sufficiently strongly to save unitarity in an evolving black hole spacetime. One limitation of the analysis in [22] is however that it was done in $1+1$ dimensions. Quantum fields generally become more singular as the spacetime dimension increases: would the conclusions in $3+1$ dimensions be similar? A second limitation is that the analysis in [22] relied on an infrared cutoff to eliminate the infrared ambiguity that the massless scalar field has in $1+1$ dimensions. Could the results in [22] be an artifact of the $(1+1)$-dimensional infrared sickness, with no counterpart in $3+1$ dimensions?

In this paper we take a first step toward adapting the wall creation analysis of [22] to $3+1$ dimensions, and answering these questions. We consider a massless scalar field in 
$(3+1)$-dimensional Minkowski spacetime, and we introduce at the spatial origin a time-dependent boundary condition that interpolates, over a finite interval of time, between ordinary Minkowski dynamics and a Dirichlettype condition. As the boundary condition is introduced at just one spatial point, the physical interpretation is now not the smooth creation of a wall but the smooth creation of a pointlike source. We then ask what happens to the energy transmitted into the field and to the response of an UnruhDeWitt detector in the limit of rapid source creation. The answers turn out to have some similarities with the $(1+1)$-dimensional analysis of [22] but also significant differences. A technical difference is that in $3+1$ dimensions there is no infrared ambiguity, and no infrared cutoff is needed. A difference in physically observable quantities is that in $3+1$ dimensions both the field's energy density and the detector's response are more singular.

First, we consider the energy. While the renormalized energy density $\left\langle T_{00}\right\rangle$ is well defined everywhere away from the source, it is bounded neither above nor below. In the outgoing pulse generated by the evolving source, $\left\langle T_{00}\right\rangle$ is unbounded below immediately to the future of the light cone of the point where the boundary condition starts to change, and the total energy in the pulse is negative infinity. After the pulse has gone, $\left\langle T_{00}\right\rangle$ is nonzero, and it diverges at $r \rightarrow 0$ proportionally to $-(\ln r) / r^{4}$ : a cloud of positive energy lingers near the source after the source is fully formed, and the total energy in this cloud is positive infinity. Further, at a fixed $r,\left\langle T_{00}\right\rangle$ is not static, and it diverges at $t \rightarrow \infty$ proportionally to $\ln t$. In the limit of rapid source creation, $\left\langle T_{00}\right\rangle$ diverges everywhere in the timelike future of the creation event. The source creation hence leaves in the late time region a large energetic memory. This memory has no counterpart in the $(1+1)$ dimensional analysis of [22].

We note that the firewall in both the previous paper [22] and the present work is not in fact modeled by the wall or point source, respectively, where the boundary conditions are specified. Instead, these serve as the source of the firewall which itself is modelled by the resulting outgoing null shell of energy. It is for this reason that it is important to calculate the response of a detector passing through the outgoing shell of energy (i.e. firewall), as we do in Sec. IV. In particular, we consider the response of a static Unruh-DeWitt detector. We find that the response of a detector that operates only in the late time region mimics $\left\langle T_{00}\right\rangle$ closely, both in the late time limit and in the limit of rapid source creation: in both limits, the response has a logarithmic divergence. We have not considered in detail the response of a detector that goes through the pulse emanating from the changing boundary condition, but the behavior in the post-pulse region is already sufficient to establish that the response does not remain finite in the limit of rapid source creation.

We conclude that the rapid creation of a source makes the $(3+1)$-dimensional field significantly more singular than the corresponding event in $1+1$ dimensions; in particular, the response of an Unruh-DeWitt detector diverges in the rapid creation limit. These results suggest that a source creation may be able to model the breaking of quantum correlations in the way that has been proposed to happen in an evolving black hole spacetime [8-14]. The persistence of large late time effects is perhaps particularly reminiscent of the energetic curtain scenario proposed in [8].

We begin in Sec. II by setting up the classical dynamics of the scalar field under the evolving boundary condition at the spatial origin. Section III introduces the quantized field and evaluates $\left\langle T_{00}\right\rangle$. The response of an Unruh-DeWitt detector is considered in Sec. IV. Section V gives a brief summary and discussion. Technical material is relegated to five appendices.

Our metric signature is mostly minus. Overline denotes complex conjugation. A continuous function of a real variable is said to be $C^{0}$, a function that is $n \in \mathbb{N}=$ $\{1,2, \ldots\}$ times continuously differentiable is said to be $C^{n}$, and a function that has all derivatives is said to be $C^{\infty}$, or smooth. We work in geometric units in which $\hbar=c=1$.

\section{CLASSICAL FIELD}

\section{A. Field equation and boundary condition}

We consider a real massless scalar field $\phi$ in $(3+1)$ dimensional Minkowski spacetime from which the spatial origin has been excised. Writing the metric as

$$
d s^{2}=d t^{2}-\left(d x^{1}\right)^{2}-\left(d x^{2}\right)^{2}-\left(d x^{3}\right)^{2},
$$

the field equation is

$$
\left(\partial_{t}^{2}-\nabla^{2}\right) \phi=0
$$

where $\nabla^{2}=\partial_{x^{1}}^{2}+\partial_{x^{2}}^{2}+\partial_{x^{3}}^{2}$. The Klein-Gordon inner product evaluated on a constant $t$ hypersurface reads

$\left(\phi_{1}, \phi_{2}\right)_{\mathrm{KG}}=i \int d x^{1} d x^{2} d x^{3}\left(\overline{\phi_{1}} \partial_{t} \phi_{2}-\left(\partial_{t} \overline{\phi_{1}}\right) \phi_{2}\right)$.

In the spherical coordinates, defined by $\left(x^{1}, x^{2}, x^{3}\right)=$ $(r \sin \theta \cos \varphi, r \sin \theta \sin \varphi, r \cos \theta)$, the metric reads

$$
d s^{2}=d t^{2}-d r^{2}-r^{2}\left(d \theta^{2}+\sin ^{2} \theta d \varphi^{2}\right)
$$

and the Klein-Gordon inner product reads

$$
\left(\phi_{1}, \phi_{2}\right)_{K G}=i \int_{0}^{\infty} r^{2} d r \int_{S^{2}} d \Omega\left(\overline{\phi_{1}} \partial_{t} \phi_{2}-\left(\partial_{t} \overline{\phi_{1}}\right) \phi_{2}\right),
$$

where $d \Omega=\sin \theta d \theta d \varphi$ is the volume element on unit $S^{2}$. The excised spatial origin is at $r=0$. 
To specify the dynamics, we need to define $\nabla^{2}$ at each $t$ as a self-adjoint operator. After decomposition into spherical harmonics, the only freedom is in the spherically symmetric sector, as discussed in Appendix A: writing

$$
\phi(t, r)=\frac{f(t, r)}{\sqrt{4 \pi} r},
$$

the eigenfunctions of $\nabla^{2}$ must satisfy the boundary condition

$$
(\cos \theta(t)) \lim _{r \rightarrow 0} f(t, r)=L(\sin \theta(t)) \lim _{r \rightarrow 0} \partial_{r} f(t, r),
$$

where $L$ is a positive constant of dimension length, introduced for dimensional convenience, and the prescribed function $\theta(t)$, taking values in $[0, \pi)$, specifies at each $t$ the self-adjoint extension of $\nabla^{2}$. We denote this extension by $\Delta_{\theta(t)}$.

$\Delta_{0}$ coincides with the unique self-adjoint extension of $\nabla^{2}$ on $L_{2}\left(\mathbb{R}^{3}\right)$, yielding usual scalar field dynamics on full Minkowski space. For $\theta \in(\pi / 2, \pi), \Delta_{\theta}$ has a positive proper eigenvalue, which on quantization would give a tachyonic instability. We therefore assume $\theta \in[0, \pi / 2]$, in which case the spectrum of $\Delta_{\theta}$ consists of the negative continuum.

We specialize to a $\theta(t)$ that interpolates between $\theta=0$ and $\theta=\pi / 2$ over a finite interval of time. We may parametrize $\theta(t)$ as

$$
\theta(t)= \begin{cases}0 & \text { for } t \leq 0, \\ \operatorname{arccot}[\lambda L \cot (h(\lambda t))] & \text { for } 0<t<\lambda^{-1}, \\ \pi / 2 & \text { for } t \geq \lambda^{-1}\end{cases}
$$

where $\lambda$ is a positive constant of dimension inverse length and $h: \mathbb{R} \rightarrow \mathbb{R}$ is a smooth function such that

$$
\begin{gathered}
h(y)=0 \quad \text { for } y \leq 0, \\
0<h(y)<\pi / 2 \quad \text { for } 0<y<1, \\
h(y)=\pi / 2 \quad \text { for } y \geq 1 .
\end{gathered}
$$

Over the interval $0<t<\lambda^{-1}$, the boundary condition (2.7) then reads

$$
\lim _{r \rightarrow 0} \frac{\partial_{r} f(t, r)}{f(t, r)}=\lambda \cot (h(\lambda t)) .
$$

In words, this parametrization means that the boundary condition interpolation takes place over time $\lambda^{-1}$ while the interpolation profile is determined by the dimensionless function $h(y)$. The limit of rapid interpolation with fixed profile is that of $\lambda \rightarrow \infty$.

\section{B. Mode functions}

As preparation for quantization, we shall write down the mode solutions that reduce to the usual Minkowski modes for $t \leq 0$. As noted above, we need consider only the spherically symmetric sector.

We work in the radial null coordinates $u:=t-r$ and $v:=t+r$, in which $t=(v+u) / 2$ and $r=(v-u) / 2$. The metric (2.4) becomes

$$
d s^{2}=d u d v-\frac{1}{4}(v-u)^{2}\left(d \theta^{2}+\sin ^{2} \theta d \varphi^{2}\right) .
$$

Taking $\phi$ to be spherically symmetric, the field equation (2.2) becomes

$$
\partial_{u} \partial_{v}(r \phi)=0 .
$$

We hence seek mode solutions with the ansatz

$$
\phi_{k}=\frac{U_{k}}{\sqrt{4 \pi} r},
$$

where

$$
U_{k}(u, v)=\frac{1}{\sqrt{4 \pi k}}\left[e^{-i k v}+E_{k}(u)\right]
$$

$k>0$, and $E_{k}$ is to be found. As any choice for $E_{k}$ satisfies the wave equation, the task is to determine $E_{k}$ so that the boundary condition (2.7) is satisfied for all $t$ and the usual Minkowski modes are obtained for $t \leq 0$.

Substituting (2.14) in the boundary condition (2.7) gives for $E_{k}$ the ordinary differential equation

$L \sin (\theta(t)) \frac{d}{d t}\left[e^{-i k t}-E_{k}(t)\right]=\cos (\theta(t))\left[e^{-i k t}+E_{k}(t)\right]$.

Writing

$$
E_{k}(u)=R_{k / \lambda}(\lambda u)
$$

and using (2.8), (2.15) takes the dimensionless form

$\sin (h(y)) \frac{d}{d y}\left[e^{-i K y}-R_{K}(y)\right]=\cos (h(y))\left[e^{-i K y}+R_{K}(y)\right]$,

where $K=k / \lambda>0$ is the dimensionless frequency and $y=\lambda u$.

To solve (2.17), we introduce the auxiliary function

$$
B(y)= \begin{cases}0 & \text { for } y \leq 0, \\ \exp \left(-\int_{y}^{1} \cot (h(z)) d z\right) & \text { for } 0<y<1, \\ 1 & \text { for } y \geq 1 .\end{cases}
$$


ZHOU, CARRINGTON, KUNSTATTER, and LOUKO

$B(y)$ is everywhere smooth: smoothness at $y=1$ follows from the smoothness of $h(z)$ near $z=1$, and smoothness at $y=0$ is shown in Appendix B. For $y>0, B(y)$ satisfies

$$
\frac{B^{\prime}(y)}{B(y)}=\cot (h(y))
$$

It follows that the solution to (2.17) is

$$
R_{K}(y)= \begin{cases}-e^{-i K y} & \text { for } y \leq 0 \\ -e^{-i K y}-\frac{2 i K}{B(y)} \int_{0}^{y} B(z) e^{-i K z} d z & \text { for } 0<y<\infty\end{cases}
$$

From (2.20) and the smoothness of $B$ we see that $R_{K}(y)$ is smooth everywhere except possibly at $y=0$, and we verify in Appendix B that $R_{K}(y)$ is $C^{25}$ at $y=0$. It follows that the mode functions are smooth everywhere except possibly at $r=t$, and they are at least $C^{25}$ at $r=t$.

An alternative expression for $R_{K}(y)$ is

$R_{K}(y)= \begin{cases}-e^{-i K y} & \text { for } y \leq 0 \\ e^{-i K y}-\frac{2}{B(y)} \int_{0}^{y} B^{\prime}(z) e^{-i K z} d z & \text { for } 0<y<1 \\ e^{-i K y}-2 C_{K} & \text { for } y \geq 1\end{cases}$

where

$$
C_{K}=\int_{0}^{1} B^{\prime}(z) e^{-i K z} d z
$$

At $u \leq 0$ and $u \geq \lambda^{-1}$, the mode functions $\phi_{k}$ (2.13) hence reduce respectively to

$$
\phi_{k}(t, r)= \begin{cases}-\frac{i e^{-i k t} \sin (k r)}{2 \pi \sqrt{k} r} & \text { for } u \leq 0 \\ \frac{e^{-i k t} \cos (k r)-C_{k / \lambda}}{2 \pi \sqrt{k} r} & \text { for } u \geq \lambda^{-1}\end{cases}
$$

For $u \leq 0, \phi_{k}(t, r)$ coincide with the usual Minkowski space mode functions. Evaluating the Klein-Gordon inner product (2.5) on a hypersurface of constant negative $t$ shows that the normalization is $\left(\phi_{k}, \phi_{k^{\prime}}\right)_{K G}=\delta\left(k-k^{\prime}\right)$. For $u \geq \lambda^{-1}$, the $r$-dependence in the numerator of $\phi_{k}(t, r)$ (2.23) contains the term $\cos (k r)$, which one would expect from the boundary condition (2.7) with $\theta=\pi / 2$, but it contains also the additive memory term $-C_{k / \lambda}$, which carries a recollection of how the boundary condition evolved from $\theta=0$ to $\theta=\pi / 2$. From (2.22) we see that $C_{K}$ is smooth in $K, C_{0}=1$, and $C_{K} \rightarrow 0$ faster than any inverse power of $K$ as $K \rightarrow \infty$, as can be verified by repeated integration by parts [26]. For fixed $\lambda$, the memory term is hence insignificant at large frequencies but
PHYSICAL REVIEW D 95, 085007 (2017)

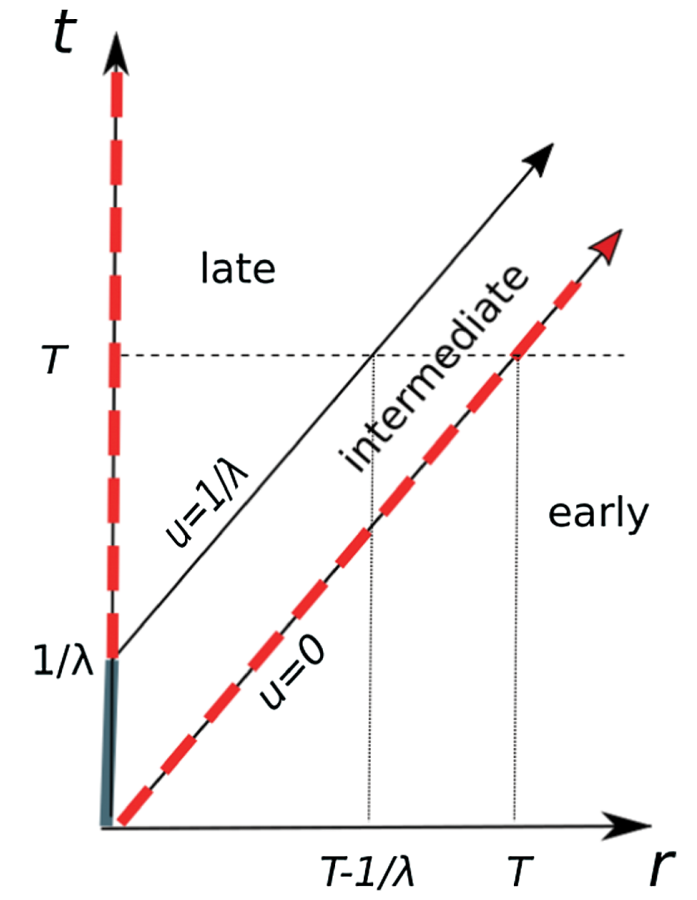

FIG. 1. Spacetime diagram of the evolving boundary condition (2.7) at $r=0$, with the angular dimensions suppressed. The interpolation between $\theta=0$ and $\theta=\pi / 2$ at $r=0$ occurs over $0<t<\lambda^{-1}$ (solid line), and the null cones of the events where the boundary condition changes fill the region $0<u<\lambda^{-1}$ in the spacetime. The early region $u<0$ is outside the null cone of $(t, r)=(0,0)$, and the mode functions there coincide with those in full Minkowski space. The mode functions in the late region $u>\lambda^{-1}$ carry a memory of the field evolution that occurred over the intermediate region $0<u<\lambda^{-1}$. The infinite contributions to the energy at $r=0$ (positive infinity) and $r=t$ (negative infinity) are shown as heavy dashed lines. The spacelike hypersurface $t=T>\lambda^{-1}$, shown as a short dashed line, intersects all three regions.

significant at low frequencies. We shall see in Sec. III that the memory term has a significant effect on the stressenergy tensor and the Wightman function.

A spacetime diagram is shown in Fig. 1, indicating the regions $u<0,0<u<\lambda^{-1}$ and $u>\lambda^{-1}$.

\section{QUANTIZED FIELD}

\section{A. Field operator and the Fock vacuum}

We quantize the field by using for the spherically symmetric sector the mode functions found in Sec. II and treating the nonzero angular momentum sectors as in ordinary Minkowski space. As we are interested in the effects due to the evolving boundary condition, compared with a field in ordinary Minkowski space, we write out only the expressions for the spherically symmetric sector.

We expand the spherically symmetric sector of the quantized field as 


$$
\phi=\int_{0}^{\infty}\left(a_{k} \phi_{k}+a_{k}^{\dagger} \bar{\phi}_{k}\right) d k,
$$

where the annihilation and creation operators have the commutators $\left[a_{k}, a_{k^{\prime}}^{\dagger}\right]=\delta\left(k-k^{\prime}\right)$. By the normalization of the mode functions, this gives the field and its time derivative the correct equal-time commutator. We denote by $|0\rangle$ the state that is annihilated by all $a_{k}$ and by all the annihilation operators of the nonzero angular momentum sectors. In the region $u<0,|0\rangle$ coincides with the usual Minkowski vacuum, which we denote by $\left|0_{M}\right\rangle$.

\section{B. Energy density}

In the Lorentz frame of the metric (2.4), the energy density of the classical scalar field is given in terms of the energy-momentum tensor by

$$
T_{00}=T_{u u}+T_{v v}+2 T_{u v},
$$

where [15]

$$
\begin{gathered}
T_{u u}=\left(\partial_{u} \phi\right)^{2}, \\
T_{v v}=\left(\partial_{v} \phi\right)^{2}, \\
T_{u v}=T_{v u}=\frac{1}{4 r^{2}}\left[\left(\partial_{\theta} \phi\right)^{2}+(\sin \theta)^{-2}\left(\partial_{\varphi} \phi\right)^{2}\right],
\end{gathered}
$$

and we have taken the scalar field to be minimally coupled. To obtain the renormalized energy density of the quantized field in the state $|0\rangle,\left\langle T_{00}\right\rangle:=\left\langle 0\left|T_{00}\right| 0\right\rangle_{\text {ren }}$, we point-split the expressions in (3.3), take the expectation value in $|0\rangle$, renormalize by subtracting the corresponding expectation value in $\left|0_{M}\right\rangle$, and finally take the coincidence limit. As $|0\rangle$ and $\left|0_{M}\right\rangle$ differ only in the spherically symmetric sector, the derivatives in (3.3c) show that $\left\langle T_{u v}\right\rangle=0$, and we find

$$
\begin{aligned}
\left\langle T_{00}\right\rangle= & \lim _{\substack{u_{1}, u_{2} \rightarrow u \\
v_{1}, v_{2} \rightarrow v}}\left(\partial_{u_{1}} \partial_{u_{2}}+\partial_{v_{1}} \partial_{v_{2}}\right)[\langle 0|\phi(1) \phi(2)| 0\rangle \\
& \left.-\left\langle 0_{M}|\phi(1) \phi(2)| 0_{M}\right\rangle\right],
\end{aligned}
$$

where $\phi$ now stands for the spherically symmetric quantum field (3.1).

To evaluate (3.4), we write $\phi$ in terms of $f$ as in (2.6). Recalling that $r=(v-u) / 2$, this gives

$$
\begin{aligned}
\left\langle T_{00}\right\rangle= & \frac{1}{4 \pi}\left[\frac{\left\langle\left(\partial_{u} f\right)^{2}\right\rangle}{r^{2}}+\frac{\left\langle\left(\partial_{v} f\right)^{2}\right\rangle}{r^{2}}\right. \\
& \left.+\frac{\left\langle f\left(\partial_{u} f-\partial_{v} f\right)\right\rangle+\left\langle\left(\partial_{u} f-\partial_{v} f\right) f\right\rangle}{2 r^{3}}+\frac{\left\langle f^{2}\right\rangle}{2 r^{4}}\right] .
\end{aligned}
$$

By (2.6), (2.13) and (3.1), $f$ has the expansion

$$
f=\int_{0}^{\infty}\left(a_{k} U_{k}+a_{k}^{\dagger} \bar{U}_{k}\right) d k .
$$

From (2.14), (2.16) and (3.6) we obtain for $\left\langle T_{00}\right\rangle$ the final expression

$$
\begin{aligned}
\left\langle T_{00}\right\rangle= & \frac{\lambda^{2}}{16 \pi^{2} r^{2}} \int_{0}^{\infty} \frac{d K}{K}\left[\left|R_{K}^{\prime}(\lambda(t-r))\right|^{2}-K^{2}\right] \\
& -\frac{1}{32 \pi^{2} r^{2}} \frac{\partial}{\partial r}\left(\frac{\mathcal{G}_{\lambda}(t, r)}{r}\right)
\end{aligned}
$$

where the prime on $R_{K}$ denotes the derivative with respect to the argument and

$$
\begin{aligned}
\mathcal{G}_{\lambda}(t, r)= & \int_{0}^{\infty} \frac{d K}{K}\left[\left|R_{K}(\lambda(t-r))\right|^{2}+2 \cos (2 K \lambda r)-1\right. \\
& \left.+R_{K}(\lambda(t-r)) e^{i K \lambda(t+r)}+\overline{R_{K}(\lambda(t-r))} e^{-i K \lambda(t+r)}\right] .
\end{aligned}
$$

The first term in (3.7) comes from the first term in (3.5), the second term in (3.7) comes from the last two terms in (3.5), and the second term in (3.5) vanishes. We note in passing that $\mathcal{G}_{\lambda}$ is related to the renormalized vacuum polarization $\left\langle\phi^{2}\right\rangle$ by

$$
\left\langle\phi^{2}\right\rangle=\frac{\mathcal{G}_{\lambda}(t, r)}{16 \pi^{2} r^{2}} .
$$

\section{Energy density in the early, late and intermediate regions}

We consider $\left\langle T_{00}\right\rangle$ separately in the early region, $t<r$, in the late region, $t>r+\lambda^{-1}$, and in the intermediate region, $r \leq t \leq r+\lambda^{-1}$.

In the early region, $t<r,|0\rangle$ coincides with $\left|0_{M}\right\rangle$, and $\left\langle T_{00}\right\rangle$ vanishes. This can be seen immediately from (3.4), and also by substituting (2.20) into (3.7) and (3.8).

In the late region, $t>r+\lambda^{-1}$, the first term in (3.7) vanishes. We show in Appendix $\mathrm{C}$ that $\left\langle T_{00}\right\rangle$ is a pointwise well defined function, it has dependence on both $t$ and $r$, it is continuous, and it has the asymptotic forms

$$
\begin{gathered}
\left\langle T_{00}\right\rangle \sim \frac{\ln t}{4 \pi^{2} r^{4}} \quad \text { as } t \rightarrow \infty \quad \text { with } \quad r \text { fixed, } \\
\left\langle T_{00}\right\rangle \sim-\frac{\ln r}{8 \pi^{2} r^{4}} \quad \text { as } r \rightarrow 0 \quad \text { with } \quad t \text { fixed. }
\end{gathered}
$$

On the hypersurface of $t=T=$ constant with $T>\lambda^{-1}$ (see Fig. 1), every ball of radius less than $T-\lambda^{-1}$ contains 
hence an infinite total energy, due to positive $\left\langle T_{00}\right\rangle$ that diverges as $r \rightarrow 0$.

In the intermediate region, $r \leq t \leq r+\lambda^{-1}$, we show in Appendix $\mathrm{D}$ that $\left\langle T_{00}\right\rangle$ is a pointwise well-defined function, and it is continuous in $r$ for $t>r$. Under the technical assumption that the third derivative of $\tan (h(y))$ is nonnegative for sufficiently small positive $y$, we show in addition that $\left\langle T_{00}\right\rangle$ is well defined also at $t=r$ (where it then vanishes); however, due to contributions from the first term in (3.7), $\left\langle T_{00}\right\rangle$ tends to negative infinity as $r \rightarrow t_{-}$, faster than any negative multiple of $1 / h(\lambda(t-r))$. In particular, $\left\langle T_{00}\right\rangle$ is not continuous at $r=t$. This implies that integrating $\left\langle T_{00}\right\rangle$ on a hypersurface of $t=T=$ constant $>0$ over an an arbitrarily small neighborhood of $r=T$ gives negative infinite energy. The changing boundary condition creates a pulse of infinite negative energy traveling outward, immediately to the future of the light cone of the point $(t, r)=(0,0)$ where the boundary condition starts to change.

Combining the results of the two previous paragraphs, it follows that the total energy on the hypersurface of $t=T=$ constant with $T>\lambda^{-1}$ is not defined, even though $\left\langle T_{00}\right\rangle$ exists at every point. Given an $r_{0} \in(0, T)$, the total energy for $r \leq r_{0}$ is positive infinite, due to a large positive contribution from $r \rightarrow 0$, while the total energy for $r \geq r_{0}$ is negative infinite, due to a large negative contribution from $r \rightarrow T_{-}$.

\section{Rapid boundary condition change}

Finally, consider the limit in which the boundary condition changes rapidly, $\lambda \rightarrow \infty$. At each given point in the region $t>r,\left\langle T_{00}\right\rangle$ diverges in this limit, with the asymptotic form

$$
\left\langle T_{00}\right\rangle \sim \frac{\ln \lambda}{8 \pi^{2} r^{4}},
$$

as we show in Appendix C. In the limit of rapid source creation, $\left\langle T_{00}\right\rangle$ hence diverges everywhere inside the light cone of the creation event. This is in a stark contrast to the corresponding $(1+1)$-dimensional wall creation, where $\left\langle T_{00}\right\rangle$ vanishes inside the light cone of the creation event [22].

\section{RESPONSE OF AN UNRUH-DEWITT DETECTOR}

In this section we consider an inertial Unruh-DeWitt (UDW) detector [24,25] at a fixed spatial location.

We consider a detector that is coupled linearly to the quantum field. Within first-order perturbation theory, the probability of the detector to undergo a transition from a state with energy 0 to a state with energy $\omega$ is proportional to the response function, given by $[15,16,24,25]$
$\mathcal{F}(\omega)=\int_{-\infty}^{\infty} d t_{1} \int_{-\infty}^{\infty} d t_{2} e^{-i \omega\left(t_{1}-t_{2}\right)} \chi\left(t_{1}\right) \chi\left(t_{2}\right) \mathcal{W}\left(t_{1}, t_{2}\right)$,

where the smooth real-valued switching function $\chi$ specifies how the detector's interaction with the field is turned on and off, and $\mathcal{W}$ is the pull-back of the field's Wightman function to the detector's worldline. In the Minkowski vacuum $\left|0_{M}\right\rangle$, we have [15]

$$
\mathcal{W}_{\left|0_{M}\right\rangle}\left(t_{1}, t_{2}\right)=-\frac{1}{4 \pi^{2}\left(t_{1}-t_{2}-i \epsilon\right)^{2}}
$$

where the limit $\epsilon \rightarrow 0_{+}$is implied and encodes the distributional part of $\mathcal{W}$, and from (4.1) we obtain $[18,27,28]$

$$
\begin{aligned}
\mathcal{F}_{\left|0_{M}\right\rangle}(\omega)= & -\frac{\omega \Theta(-\omega)}{2 \pi} \int_{-\infty}^{\infty} d u[\chi(u)]^{2} \\
& +\frac{1}{2 \pi^{2}} \int_{0}^{\infty} d s \frac{\cos (\omega s)}{s^{2}} \\
& \times \int_{-\infty}^{\infty} d u \chi(u)[\chi(u)-\chi(u-s)],
\end{aligned}
$$

where $\Theta$ is the Heaviside function. Denoting by $\mathcal{F}_{|0\rangle}$ the response function in the state $|0\rangle$, and setting $\Delta \mathcal{F}=\mathcal{F}_{|0\rangle}-\mathcal{F}_{\left|0_{M}\right\rangle}$, we then have

$\Delta \mathcal{F}(\omega)=\int_{-\infty}^{\infty} d t_{1} \int_{-\infty}^{\infty} d t_{2} e^{-i \omega\left(t_{1}-t_{2}\right)} \chi\left(t_{1}\right) \chi\left(t_{2}\right) \Delta \mathcal{W}\left(t_{1}, t_{2}\right)$,

where

$$
\begin{aligned}
\Delta \mathcal{W}\left(t_{1}, t_{2}\right)= & \frac{1}{4 \pi r^{2}} \int_{0}^{\infty}\left(U_{k}\left(t_{1}-r, t_{1}+r\right) \overline{U_{k}\left(t_{2}-r, t_{2}+r\right)}\right. \\
& \left.-U_{k}^{M}\left(t_{1}-r, t_{1}+r\right) \overline{U_{k}^{M}\left(t_{2}-r, t_{2}+r\right)}\right) d k,
\end{aligned}
$$

$r$ is the location of the detector, and $U^{M}$ is as in (2.14) but with $E_{k}(u)=-e^{-i k u}$ for all $u$. Note that $\Delta \mathcal{W}\left(t_{1}, t_{2}\right)$ vanishes when $t_{1}, t_{2} \leq r$.

We consider a detector that operates only in the future region, $t>r+\lambda^{-1}$. For $t_{1}, t_{2}>r+\lambda^{-1}$, the integrand in (4.5) can be rearranged and split to give 


$$
\begin{aligned}
4 \pi^{2} r^{2} \Delta \mathcal{W}\left(t_{1}, t_{2}\right)= & \int_{0}^{\infty} \frac{d K}{K}\left[\left(1-C_{K}\right) e^{i K \lambda t_{2}}+\left(1-\bar{C}_{K}\right) e^{-i K \lambda t_{1}}\right] \cos (K \lambda r)+\int_{0}^{\infty} \frac{d K}{K}\left[\left|C_{K}\right|^{2}-\cos (K \lambda r)\right] \\
& +\int_{0}^{\infty} \frac{d K}{K}\left[\left(1-e^{i K \lambda t_{2}}\right)+\left(1-e^{-i K \lambda t_{1}}\right)\right] \cos (K \lambda r)+\int_{0}^{\infty} \frac{d K}{K}\left(e^{-i K \lambda\left(t_{1}-t_{2}\right)}-1\right) \cos (2 K \lambda r) \\
& +\int_{0}^{\infty} \frac{d K}{K}[\cos (2 K \lambda r)-\cos (K \lambda r)] .
\end{aligned}
$$

The integrals can be evaluated by the formulas of Appendix E, with the result

$$
\begin{aligned}
8 \pi^{2} r^{2} \Delta \mathcal{W}\left(t_{1}, t_{2}\right)= & H\left(\lambda\left(t_{2}+r\right)\right)+H\left(\lambda\left(t_{2}-r\right)\right)+H\left(\lambda\left(t_{1}+r\right)\right)+H\left(\lambda\left(t_{1}-r\right)\right)+\ln \left(\frac{\lambda^{2}\left(t_{1}^{2}-r^{2}\right)\left(t_{2}^{2}-r^{2}\right)}{\left|4 r^{2}-\left(t_{1}-t_{2}\right)^{2}\right|}\right) \\
& +i \pi\left[\Theta\left(t_{2}-t_{1}-2 r\right)-\Theta\left(t_{1}-t_{2}-2 r\right)\right]+2 k_{1},
\end{aligned}
$$

where the function $H$ is defined in Proposition E.2 and the constant $k_{1}$ is given by (E.2). Note that $\mathcal{W}\left(t_{1}, t_{2}\right)$ has singularities at $\left|t_{1}-t_{2}\right|=2 r$, which is when the two points are separated by a null geodesic that bounces off the origin, but this singularity is only logarithmic, and $\Delta \mathcal{W}\left(t_{1}, t_{2}\right)$ is representable by a function. Note also that the first four terms in (4.7) are real because $t_{1}, t_{2}>r+\lambda^{-1}$ by assumption and $H(\alpha)$ is real for $\alpha \geq 1$ by (E.4).

We consider two limits.

First, suppose that the support of $\chi$ is contained in some finite interval of fixed length, centered at $t=t_{c}$, and consider the limit $t_{c} \rightarrow \infty$. By the large argument expansion of $H$ in (E.5), the contribution from the $H$-terms in (4.7) vanishes in this limit, and we have

$$
\Delta \mathcal{F}(\omega) \sim \frac{\left(\ln t_{c}\right)|\hat{\chi}(\omega)|^{2}}{2 \pi^{2} r^{2}}
$$

where the hat denotes the Fourier transform, $\hat{\chi}(\omega):=$ $\int_{-\infty}^{\infty} e^{-i \omega t} \chi(t) d t . \Delta \mathcal{F}$ hence diverges in this limit, proportionally to $\ln t_{c}$. This is similar to the late time divergence of $\left\langle T_{00}\right\rangle$ (3.10a).

Second, consider the limit of large $\lambda$. We assume that the support of $\chi$ is contained in $[r+a, \infty)$, where $a$ is a positive constant, and we take $\lambda$ large enough that $\lambda^{-1}<a$. By similar arguments, we find

$$
\Delta \mathcal{F}(\omega)=\frac{(\ln \lambda)|\hat{\chi}(\omega)|^{2}}{4 \pi^{2} r^{2}}+O(1) .
$$

The $\ln \lambda$ divergence in (4.9) at $\lambda \rightarrow \infty$ is similar to the $\ln \lambda$ divergence of $\left\langle T_{00}\right\rangle$ in (3.11).

\section{SUMMARY AND DISCUSSION}

We have addressed the smooth and sharp creation of a pointlike source for a massless scalar field in $(3+1)$ dimensional Minkowski spacetime, implemented by introducing at the spatial origin a time-dependent boundary condition that interpolates between ordinary Minkowski dynamics and a Dirichlet-type boundary condition. We found that the process is significantly more singular than a corresponding creation of a wall in $(1+1)$-dimensional Minkowski spacetime [22]. While $\left\langle T_{00}\right\rangle$ is well defined away from the source, it is unbounded from above and below: there is a pulse of infinite negative energy traveling outward, and there is a cloud of infinite positive energy that lingers around the fully formed source. In the rapid source creation limit, $\left\langle T_{00}\right\rangle$ diverges everywhere in the timelike future of the creation event, and so does the response of an Unruh-DeWitt detector that operates in the timelike future of the creation event.

There are two technical reasons for the differences between our $(3+1)$-dimensional process and the corresponding $(1+1)$-dimensional process analyzed in [22]. First, as our boundary condition is at a single spatial point, it does not divide the $(3+1)$-dimensional spacetime into two regions. Our boundary condition in fact resembles more closely the removal of a $(1+1)$-dimensional wall than its creation [23]. This affects both $\left\langle T_{00}\right\rangle$ and the response of the Unruh-DeWitt detector. Second, the $(3+1)$-dimensional $\left\langle T_{00}\right\rangle$ (3.5) contains terms that have no counterpart in $1+1$ dimensions, and these additional terms are especially significant near the source.

We emphasize that the infinite negative energy radiating from the evolving source is localized in the immediate future of the light cone of the point where the boundary condition starts to change, and this negative energy cannot be made finite by slowing down the boundary condition change. We have verified, adapting the methods of our Appendix D and under analogous technical assumptions, that a similar infinite energy occurs also in the $(1+1)$ dimensional wall creation of Sec. II in [22], but with two qualitative differences: the infinite energy in [22] is localized not where the boundary condition starts to change but where the boundary condition approaches its final value, and the infinite energy has positive sign. Specifically, formula (2.17b) in [22] tends to $+\infty$ as $u \rightarrow \lambda_{-}^{-1}$, so fast that the total energy in (2.18) and (2.19) is positive infinity. 
Formula (2.20) in [22] is hence not correct: the term denoted therein by $O(1)$ should be replaced by positive infinity. We suspect that similar comments may apply to formulas (3.7b), (3.8) and (3.9) in [22]. Note, however, that the results about detector response versus total energy in [22] were obtained via the boundary condition family (4.1), and they are hence not affected by the infinities that occur in (2.18)-(2.20).

Our results, including the divergent negative energy near $r=t$, suggest that the creation of a pointlike source in quantum field theory may be sufficiently singular to model the breaking of correlations that has been proposed to happen at the horizon of an evaporating black hole [8-14]. It is conceivable that the divergent negative energy near $r=t$ and the divergent positive energy near $r=0$ could be arranged to cancel and produce a finite total energy on each hypersurface of constant $t$, but such a cancellation would require a nonlocal correlation between the regulator near $r=t$ and the regulator near $r=0$.

We note in passing that while the source creation contributes to the imaginary part of the Wightman function, the imaginary part of the Wightman function on a trajectory of constant $r$ in the late time region consists only of the terms proportional to $\Theta\left(t_{2}-t_{1}-2 r\right)$ and $\Theta\left(t_{1}-t_{2}-2 r\right)$ in (4.7). As the imaginary part of the Wightman function is the commutator, this shows that the source creation does not produce a lingering violation of strong Huygens' principle in the late time region on a trajectory of constant $r$. The source creation does hence not appear to offer opportunities for enhanced quantum communication of the kind examined in [29-31].

Finally, we anticipate that our techniques can be adapted to address an evolving boundary condition on a spherical shell or ball, where the dynamics will be potentially more germane for modeling possible new physics in the spacetime of an evaporating black hole. In particular, will the evolving boundary condition on the spherical shell or ball lead to diverging positive or negative energies in some regions of the spacetime?

\section{ACKNOWLEDGMENTS}

We thank Jim Langley for providing the proof of Proposition B.1, Eduardo Martín-Martínez for raising the question of the strong Huygens' principle violation, and Joel Feinstein and Alex Schenkel for helpful discussions. This work was funded in part by the Natural Sciences and Engineering Research Council of Canada (M.E. C. and G. K.) and by Science and Technology Facilities Council (J.L., Theory Consolidated Grant ST/J000388/1). For hospitality, G. K. thanks the University of Nottingham, and J. L. thanks the University of Winnipeg, the Winnipeg Institute for Theoretical Physics, and the Nordita 2016 "Black Holes and Emergent Spacetime" program.

\section{APPENDIX A: SCALAR LAPLACIAN ON PUNCTURED $\mathbb{R}^{n}$}

In this appendix we record relevant properties of the scalar Laplacian on punctured Euclidean $\mathbb{R}^{n}$ with $n \geq 2$.

We use spherical coordinates in which $r$ is the radial coordinate and the puncture is at $r=0$. The scalar Laplacian reads

$$
\nabla^{2}=\frac{1}{r^{n-1}} \partial_{r}\left(r^{n-1} \partial_{r}\right)+\frac{1}{r^{2}} \nabla_{S^{n-1}}^{2},
$$

where $\nabla_{S^{n-1}}^{2}$ is the Laplacian on unit $S^{n-1}$. The $L_{2}$ inner product is

$$
\left(g_{1}, g_{2}\right)=\int_{0}^{\infty} r^{n-1} d r \int_{S^{n-1}} d \Omega \overline{g_{1}} g_{2},
$$

where $d \Omega$ is the volume element on unit $S^{n-1}$.

The scaling $g=r^{(1-n) / 2} f$ maps the inner product to

$$
\left(f_{1}, f_{2}\right)_{s c}=\int_{0}^{\infty} d r \int_{S^{n-1}} d \Omega \overline{f_{1}} f_{2}
$$

and $\nabla^{2}$ to

$$
\nabla_{s c}^{2}=\partial_{r}^{2}-\frac{(n-1)(n-3)}{4 r^{2}}+\frac{1}{r^{2}} \nabla_{S^{n-1}}^{2} .
$$

After decomposition into spherical harmonics, $\nabla_{s c}^{2}$ reduces for each harmonic to the operator $\partial_{r}^{2}-a / r^{2}$, where $a \geq-1 / 4$, and the inner product $(\cdot, \cdot)_{s c}$ reduces to the standard $L_{2}$ inner product on the positive half-line. The self-adjoint extensions of $\nabla_{s c}^{2}$ for each harmonic can hence be analyzed by standard methods $[32,33]$ (for a pedagogical introduction see [34]), and the outcomes are summarized in [35]. The self-adjoint extension is unique except for $a=-1 / 4$, which occurs in the spherically symmetric sector for $n=2$, and for $a=0$, which occurs in the spherically symmetric sector for $n=3$. In each of these two cases there is a $U(1)$ family of self-adjoint extensions, characterized by a boundary condition at the origin.

In the $n=3$ spherically symmetric sector, the boundary condition at the origin is

$$
\cos \theta \lim _{r \rightarrow 0} f(r)=L \sin \theta \lim _{r \rightarrow 0} f^{\prime}(r),
$$

where $L$ is a positive constant of dimension length, introduced for dimensional convenience, and $\theta \in[0, \pi)$ is the parameter that specifies the extension. For $\theta \in$ $[0, \pi / 2]$ the spectrum consists of the negative continuum, while for $\theta \in(\pi / 2, \pi)$ there is also one proper eigenvalue, which is positive and nondegenerate. The case $\theta=0$ reduces to the essentially self-adjoint operator $\nabla^{2}$ on $L_{2}\left(\mathbb{R}^{3}\right)$. 


\section{APPENDIX B: MODE FUNCTION REGULARITY ACROSS $r=t$}

In this appendix we show that the function $B(y)(2.18)$ is smooth at $y=0$ and the function $R_{K}(y)(2.20)$ is $C^{25}$ at $y=0$. This shows that the mode functions are $C^{25}$ across $r=t$.

\section{1. $B(y)(2.18)$}

We shall show that the function $B(y)$ (2.18) is smooth at $y=0$.

From (2.18) it is immediate that $B(y) \rightarrow 0$ as $y \rightarrow 0_{+}$. We show below in Proposition B.1 that $B^{(n)}(y) \rightarrow 0$ as $y \rightarrow 0_{+}$for $n \in \mathbb{N}=\{1,2, \ldots\}$. From this it follows by L'Hôpital and induction in $n$ that all derivatives of $B(y)$ at $y=0$ exist and vanish.

Proposition B.1. For $n \in \mathbb{N}, B^{(n)}(y) \rightarrow 0$ as $y \rightarrow 0_{+}$.

Proof.-(This proof was provided by Jim Langley.) Let $0<y<1$, and write $g(y):=\tan (h(y))$, where $h$ was defined in Sec. II A. Note that $g(y)>0, g(y)$ and all its derivatives approach 0 as $y \rightarrow 0_{+}$, and from (2.18) we have

$$
\begin{gathered}
B(y)=\exp \left(-\int_{y}^{1} \frac{d z}{g(z)}\right), \\
B^{\prime}(y)=B(y) / g(y) .
\end{gathered}
$$

For $n \in \mathbb{N}$, induction gives

$$
\begin{gathered}
B^{(n)}(y)=P_{n}(y) f_{n}(y), \\
f_{n}(y)=\frac{B(y)}{(g(y))^{n}},
\end{gathered}
$$

where each $P_{n}$ is a polynomial in $g$ and its derivatives. Since each $P_{n}$ is bounded as $y \rightarrow 0_{+}$, it suffices to show that $f_{n}(y) \rightarrow 0$ as $y \rightarrow 0_{+}$for $n \in \mathbb{N}$.

From (B3b) we have

$$
\ln \left(f_{n}(y)\right)=-\left(\int_{y}^{1} \frac{d z}{g(z)}\right)\left(1+\frac{n \ln (g(y))}{\int_{y}^{1} \frac{d z}{g(z)}}\right) .
$$

As $y \rightarrow 0_{+}$, the first parentheses in (B4) tend to $\infty$, while the second parentheses tend to 1 by L'Hôpital. Hence $\ln \left(f_{n}(y)\right) \rightarrow-\infty$ as $y \rightarrow 0_{+}$, by which $f_{n}(y) \rightarrow 0$ as $y \rightarrow 0_{+}$.

\section{2. $\boldsymbol{R}_{K}(\boldsymbol{y})(2.20)$}

We shall show that the function $R_{K}(y)(2.20)$ is $C^{25}$ at $y=0$.

We write (2.20) as

$$
R_{K}(y)= \begin{cases}-e^{-i K y} & \text { for } y \leq 0, \\ -e^{-i K y}-2 i K S_{K}(y) & \text { for } 0<y<\infty,\end{cases}
$$

where $K>0$ and

$$
\begin{gathered}
S_{K}(y)=J_{K}(y) / B(y), \\
J_{K}(y)=\int_{0}^{y} B(z) e^{-i K z} d z .
\end{gathered}
$$

We show below in Proposition B.3 that $S_{K}^{(n)}(y) \rightarrow 0$ as $y \rightarrow 0_{+}$for $n=0,1,2, \ldots, 25$. This and (B5) show that $R_{K}(y)$ is $C^{25}$ at $y=0$. For the purposes of Appendix D, we formulate Proposition B.3 for $S_{K}$ that is defined by (B6) not just for $K>0$ but for $K \in \mathbb{R}$.

Lemma B.2. For $K \in \mathbb{R}, 0<y<1$ and $n \in\{1,2, \ldots, 25\}$, we have

$$
S_{K}^{(n)}(y)=\frac{h_{K, n}(y)}{B(y)(g(y))^{n}},
$$

where $g$ was defined above (B1) and $h_{K, n}$ satisfies

$h_{K, n}^{(k)}(y)=r_{K, n, k}(y) B(y)+s_{K, n, k}(y) J_{K}(y) \quad$ for $0 \leq k \leq n$,

where each $r_{K, n, k}$ and $s_{K, n, k}$ is a polynomial in $g$, its derivatives and $e^{-i K y}$, and $r_{K, n, n}(y) \rightarrow 0$ as $y \rightarrow 0_{+}$.

Proof.-Starting from (B6) and using repeatedly (B2) and the identity

$$
J_{K}^{\prime}(y)=e^{-i K y} B(y),
$$

we have verified the claim case by case for each $n$ and $k$, with the help of algebraic computing.

Proposition B.3. For $K \in \mathbb{R}$ and $n \in\{0,1,2, \ldots, 25\}$, $S_{K}^{(n)}(y) \rightarrow 0$ as $y \rightarrow 0_{+}$.

Proof.-Consider $S_{K}$. We use in (B6a) L'Hôpital with (B2) and (B9), obtaining $\lim _{y \rightarrow 0_{+}} S(y)=\lim _{y \rightarrow 0_{+}} J^{\prime}(y) /$ $B^{\prime}(y)=\lim _{y \rightarrow 0_{+}} e^{-i K y} g(y)=0$.

Consider then the derivatives of $S_{K}$. From (B2) we have

$$
\frac{d}{d y}\left[B(y)(g(y))^{n}\right]=B(y)(g(y))^{n-1}\left(1+n g^{\prime}(y)\right) .
$$

By Lemma B.2, we may hence evaluate $\lim _{y \rightarrow 0_{+}} S_{K}^{(n)}(y)$ for $n \geq 1$ by applying L'Hôpital to (B7) $n$ times, using after the $n$th differentiation $\lim _{y \rightarrow 0_{+}} J_{K}(y) / B(y)=$ $\lim _{y \rightarrow 0_{+}} S_{K}(y)=0$.

We stopped Lemma B. 2 at $n=25$ because of computing time limitations in the case-by-case proof. If Lemma B.2 extends to $n \in \mathbb{N}$, the proof of Proposition B.3 generalizes to $n \in \mathbb{N}$ and implies smoothness of $R_{K}(y)$ at $y=0$. 


\section{APPENDIX C: $\left\langle T_{00}\right\rangle$ AT LATE TIMES}

In this appendix we verify the properties of $\left\langle T_{00}\right\rangle$ quoted in Secs. III C and III D in the late time region, $t>r+\lambda^{-1}$.

Let $t>r+\lambda^{-1}$. From the last line of (2.21) we see that the first term in (3.7) vanishes. It hence suffices to consider $\mathcal{G}_{\lambda}$ (3.8), which by the last line of (2.21) reduces to

$$
\begin{aligned}
\mathcal{G}_{\lambda}(t, r)= & 4 \int_{0}^{\infty} \frac{d K}{K}\left[\left|C_{K}\right|^{2}+\cos (2 K \lambda r)\right. \\
& \left.-\left(C_{K} e^{i K \lambda t}+\bar{C}_{K} e^{-i K \lambda t}\right) \cos (K \lambda r)\right],
\end{aligned}
$$

where the integral is convergent (at large $K$ in the sense of an improper Riemann integral) by the properties of $C_{K}$ noted in Sec. II B: $C_{K}$ is smooth in $K, C_{0}=1$, and $C_{K} \rightarrow 0$ faster than any inverse power of $K$ as $K \rightarrow \infty$.

Rearranging the integrand in $(\mathrm{C} 1)$ gives

$$
\begin{aligned}
\mathcal{G}_{\lambda}(t, r)= & 4 \int_{0}^{\infty} \frac{d K}{K}\left[\left(1-C_{K}\right) e^{i K \lambda t}+\left(1-\bar{C}_{K}\right) e^{-i K \lambda t}\right] \\
& \times \cos (K \lambda r) \\
& +2 \int_{0}^{\infty} \frac{d K}{K}\left[\left|C_{K}\right|^{2}-\cos (K \lambda(t+r))\right] \\
& +2 \int_{0}^{\infty} \frac{d K}{K}\left[\left|C_{K}\right|^{2}-\cos (K \lambda(t-r))\right] \\
& +2 \int_{0}^{\infty} \frac{d K}{K}[\cos (2 K \lambda r)-\cos (K \lambda(t+r))] \\
& +2 \int_{0}^{\infty} \frac{d K}{K}[\cos (2 K \lambda r)-\cos (K \lambda(t-r))] .
\end{aligned}
$$

The integrals can be evaluated by the formulas of Appendix E, with the result

$$
\begin{aligned}
\mathcal{G}_{\lambda}(t, r)= & 2 H(\lambda(t+r))+2 H(\lambda(t-r)) \\
& +2 H(\lambda(t+r))+2 H(\lambda(t-r)) \\
& +4 \ln \left(\frac{\lambda\left(t^{2}-r^{2}\right)}{r}\right)-4 \ln 2+4 k_{1},
\end{aligned}
$$

where the function $H$ is defined in Proposition E.3 and the constant $k_{1}$ is given by (E2).

The observations in Secs. III C and III D about $\left\langle T_{00}\right\rangle$ at $t>r+\lambda^{-1}$ follow from (C3) by Proposition E.2.

\section{APPENDIX D: $\left\langle T_{00}\right\rangle$ AT INTERMEDIATE TIMES}

In this appendix we verify the properties of $\left\langle T_{00}\right\rangle$ quoted in Sec. IIIC in the intermediate time region, $r \leq t \leq r+\lambda^{-1}$.

\section{Preliminaries}

For $r<t<r+\lambda^{-1}$, the integrals in (3.8) and in the first term in (3.7) are convergent because (2.21) implies for fixed $y \in(0,1)$ the small $K$ estimates

$$
\begin{gathered}
R_{K}(y)=-1+O(K), \\
\left|R_{K}^{\prime}(y)\right|^{2}=O\left(K^{2}\right),
\end{gathered}
$$

and the large $K$ estimates

$$
\begin{gathered}
R_{K}(y)=e^{-i K y}\left[1+2 \frac{B^{\prime}(y)}{B(y)} \frac{1}{i K}+O\left(K^{-2}\right)\right], \\
\left|R_{K}(y)\right|^{2}=1+O\left(K^{-2}\right), \\
\left|R_{K}^{\prime}(y)\right|^{2}=K^{2}+O\left(K^{-2}\right) .
\end{gathered}
$$

For $t=r$, the integrands in (3.8) and in the first term of (3.7) vanish.

For $t=r+\lambda^{-1}$, the integrand in (3.7) vanishes, while (3.8) is given by (C1) with $t=r+\lambda^{-1}$, and all the steps from (C1) to (C3) still hold with $t=r+\lambda^{-1}$.

Collecting, we see that $\mathcal{G}_{\lambda}(t, r)$ (3.8) and the first term in (3.7) are well defined everywhere in $r \leq t \leq r+\lambda^{-1}$.

What remains is to examine the existence and continuity of $\partial_{r} \mathcal{G}_{\lambda}(t, r)$, and the continuity of the first term in (3.7). We address each in turn.

\section{2. $\partial_{r} \mathcal{G}_{\lambda}(t, r)$}

We show first that $\partial_{r} \mathcal{G}_{\lambda}(t, r)$ exists and is continuous in $r$ for $0<r<t$, for each positive $t$. We then assume that $g^{\prime \prime \prime}(y) \geq 0$ for sufficiently small positive $y$, and show that $\partial_{r} \mathcal{G}_{\lambda}(t, r) \rightarrow 0$ as $r \rightarrow t_{-}$. This establishes that the second term in (3.7) exists and is continuous in $r$.

We introduce dimensionless variables by $\lambda t=\sigma>0$ and $\lambda r=\sigma-y$, where $0<y<\sigma$. The quantity of interest is then $\mathcal{G}_{\lambda}(\sigma / \lambda,(\sigma-y) / \lambda)=F_{-}(y)+F_{+}(y)$, where

$$
\begin{aligned}
F_{-}(y)= & \int_{0}^{1} \frac{d K}{K}\left[\left|R_{K}(y)\right|^{2}+2 \cos (2 K(\sigma-y))-1\right. \\
& \left.+R_{K}(y) e^{i K(2 \sigma-y)}+\overline{R_{K}(y)} e^{-i K(2 \sigma-y)}\right]
\end{aligned}
$$

$$
\begin{aligned}
F_{+}(y)= & \int_{1}^{\infty} \frac{d K}{K}\left[\left|R_{K}(y)\right|^{2}+2 \cos (2 K(\sigma-y))-1\right. \\
& \left.+R_{K}(y) e^{i K(2 \sigma-y)}+\overline{R_{K}(y)} e^{-i K(2 \sigma-y)}\right],
\end{aligned}
$$

and the notation suppresses the dependence of $F_{ \pm}$on $\sigma$.

In $F_{-}$, using (B5) gives

$$
\begin{aligned}
F_{-}(y)= & 2 \int_{0}^{1} d K\left[i\left(e^{i K y}-e^{i K(2 \sigma-y)}\right) S_{K}(y)\right. \\
& \left.-i\left(e^{-i K y}-e^{-i K(2 \sigma-y)}\right) \overline{S_{K}(y)}+2\left|S_{K}(y)\right|^{2}\right] .
\end{aligned}
$$

Straightforward convergence estimates show that $F_{-}(y)$ is $C^{1}$ for $y>0$, and estimates using Proposition B. 3 show that $F_{-}^{\prime}(y) \rightarrow 0$ as $y \rightarrow 0$. 
In $F_{+}$, we use the identity

$$
R_{K}(y)=e^{-i K y}-\frac{2 i}{K}\left[\frac{B^{\prime}(y)}{B(y)} e^{-i K y}-V_{K}(y)\right],
$$

where

$$
V_{K}(y)=\frac{1}{B(y)} \int_{0}^{y} B^{\prime \prime}(z) e^{-i K z} d z
$$

obtained by integrating (2.21) by parts. This gives

$$
\begin{aligned}
F_{+}(y)= & 2 \int_{1}^{\infty} d K\left\{\frac{2}{K^{3}}\left(\frac{B^{\prime}(y)}{B(y)}\right)^{2}+\frac{2}{K} \cos (2 K(\sigma-y))+\frac{2}{K^{2}} \frac{B^{\prime}(y)}{B(y)} \sin (2 K(\sigma-y))\right. \\
& \left.+\left[-\frac{2}{K^{3}} \frac{B^{\prime}(y)}{B(y)} e^{i K y}+\frac{i}{K^{2}} e^{i K y}+\frac{i}{K^{2}} e^{i K(2 \sigma-y)}\right] V_{K}(y)+\left[-\frac{2}{K^{3}} \frac{B^{\prime}(y)}{B(y)} e^{-i K y}-\frac{i}{K^{2}} e^{-i K y}-\frac{i}{K^{2}} e^{-i K(2 \sigma-y)}\right] \frac{V_{K}(y)}{K^{3}}\left|V_{K}(y)\right|^{2}\right\},
\end{aligned}
$$

from which straightforward estimates show that $F_{+}(y)$ is $C^{1}$ for $y>0$.

To examine $F_{+}(y)$ and $F_{+}^{\prime}(y)$ as $y \rightarrow 0$, we evaluate the integral over $K$ in (D7). In the terms that do not involve $V_{K}$, the integral over $K$ produces elementary functions and the cosine integral $\mathrm{Ci}$ [36]. In the terms that involve $V_{K}$, we use (D6), we interchange the integrations as justified by the absolute convergence of the multiple integral, and we evaluate first the integral over $K$ in terms of elementary functions and the exponential integral $E_{1}$ [36]. Among the terms that ensue, several have $B^{\prime}$ or $B^{\prime \prime}$ under an integral; however, integration by parts reduces most of these terms to combinations that involve $S_{1}(y)$ and $T_{1}(y)$, where

$$
T_{K}(y)=\frac{1}{B(y)} \int_{0}^{y} B(z) z e^{-i K z} d z,
$$

and the small $y$ behavior of these terms and their derivatives can be analyzed by Proposition B.3 and its generalizations. We find that $F_{+}$decomposes as $F_{+}(y)=$ $F_{+1}(y)+F_{+2}(y)$, where we omit the lengthy expression for $F_{+1}(y)$ but just note that it satisfies $F_{+1}(y) \rightarrow 0$ and $F_{+1}^{\prime}(y) \rightarrow 0$ as $y \rightarrow 0$, while the expression for $F_{+2}(y)$ for $y<1$ reads

$$
\begin{aligned}
F_{+2}(y)= & \frac{4}{B^{2}(y)} \int_{0}^{y} d z B^{\prime}(z) \\
& \times \int_{0}^{z} d t \cos t B^{\prime}(z-t) \frac{g(z)-g(z-t)}{t} .
\end{aligned}
$$

To control $F_{+2}(y)$, we introduce the additional technical assumption that $g^{\prime \prime \prime}(y) \geq 0$ for sufficiently small positive $y$.
For sufficiently small positive $y$, an elementary analysis then gives for $t \in[0, y]$ the inequalities

$$
\begin{aligned}
\frac{g^{\prime}(y)}{y} & \leq \frac{g^{\prime}(y)-g^{\prime}(y-t)}{t} \leq g^{\prime \prime}(y), \\
\frac{g(y)}{y} & \leq \frac{g(y)-g(y-t)}{t} \leq g^{\prime}(y),
\end{aligned}
$$

understood at $t=0$ in the limiting sense. From now on we assume $y<1$ and so small that (D10) hold.

Consider now $F_{+2}(y)$. Applying L'Hôpital in (D9) and using (D10b), we find that $F_{+2}(y) \rightarrow 0$ as $y \rightarrow 0$.

Consider then $F_{+2}^{\prime}(y)$. Differentiating (D9) gives

$$
\begin{aligned}
F_{+2}^{\prime}(y)= & \frac{4}{g(y) B^{2}(y)}\left[B(y) \int_{0}^{y} d t \cos t B^{\prime}(y-t) \frac{g(y)-g(y-t)}{t}\right. \\
& \left.-2 \int_{0}^{y} d z B^{\prime}(z) \int_{0}^{z} d t \cos t B^{\prime}(z-t) \frac{g(z)-g(z-t)}{t}\right] .
\end{aligned}
$$

For the limit of $F_{+2}^{\prime}(y)$ as $y \rightarrow 0$, L'Hôpital shows that it suffices to consider

$$
\begin{aligned}
& \frac{2}{g(y) B(y)} \int_{0}^{y} d t \cos t\left[-B^{\prime}(y-t) \frac{g(y)-g(y-t)}{t}\right. \\
& \quad+g(y) B^{\prime \prime}(y-t) \frac{g(y)-g(y-t)}{t} \\
& \left.\quad+g(y) B^{\prime}(y-t) \frac{g^{\prime}(y)-g^{\prime}(y-t)}{t}\right] .
\end{aligned}
$$


The last term in (D12) can be controlled by (D10a). The combination of the first two terms can be controlled by taking $y$ to be so small that $g^{\prime}<1$, writing $B^{\prime}=g B^{\prime \prime} /\left(1-g^{\prime}\right)$, and using (D10b) and the monotonicity of $g^{\prime}$. We find that $F_{+2}^{\prime}(y) \rightarrow 0$ as $y \rightarrow 0$.

Combining these results shows that $\partial_{r} \mathcal{G}_{\lambda}(t, r)$ is continuous in $r$ for $0<r \leq t$. This establishes that the second term in (3.7) exists at each point and is continuous in $r$.

\section{3. (3.7) first term}

To analyse the first term in (3.7), it suffices to consider $\tilde{F}(y)=\tilde{F}_{-}(y)+\tilde{F}_{+}(y)$, where $y>0$ and

$$
\begin{aligned}
& \tilde{F}_{-}(y)=\int_{0}^{1} \frac{d K}{K}\left[\left|R_{K}^{\prime}(y)\right|^{2}-K^{2}\right], \\
& \tilde{F}_{+}(y)=\int_{1}^{\infty} \frac{d K}{K}\left[\left|R_{K}^{\prime}(y)\right|^{2}-K^{2}\right] .
\end{aligned}
$$

We show first that $\tilde{F}(y)$ is continuous for $y>0$. We then assume that $g^{\prime \prime \prime}(y) \geq 0$ for sufficiently small positive $y$, and show that $\tilde{F}(y) \rightarrow-\infty$ as $y \rightarrow 0$, faster than any negative multiple of $1 / g(y)$.

In $\tilde{F}_{-}$, we use (B5) and proceed as with $F_{-}$(D3). We find that $\tilde{F}_{-}(y)$ is continuous for $y>0$ and $\tilde{F}_{-}(y) \rightarrow 0$ as $y \rightarrow 0$.

In $\tilde{F}_{+}$, we start as with $F_{+}$(D3b), finding

$$
\begin{aligned}
\tilde{F}_{+}(y)= & 2 \int_{1}^{\infty} d K\left\{\frac{2}{K^{3}}\left(\frac{B^{\prime}(y)}{B(y)}\right)^{4}+\frac{2}{K^{3}}\left(\frac{B^{\prime}(y)}{B(y)}\right)^{2}\left|V_{K}(y)\right|^{2}\right. \\
& -\frac{2}{K^{3}}\left(\frac{B^{\prime}(y)}{B(y)}\right)^{3}\left[e^{i K y} V_{K}(y)+e^{-i K y} \overline{V_{K}(y)}\right] \\
& +\frac{2 i}{K^{2}}\left(\frac{B^{\prime}(y)}{B(y)}\right)^{2}\left[e^{i K y} V_{K}(y)-e^{-i K y} \overline{V_{K}(y)}\right] \\
& \left.-\frac{i}{K^{2}} \frac{B^{\prime}(y)}{B(y)}\left[e^{i K y} W_{K}(y)-e^{-i K y} \overline{W_{K}(y)}\right]\right\}, \quad \text { (D15) }
\end{aligned}
$$

where $V_{K}$ is given by (D6) and

$$
W_{K}(y)=\frac{1}{B(y)} \int_{0}^{y} B^{\prime \prime \prime}(z) e^{-i K z} d z
$$

This shows that $\tilde{F}_{+}(y)$ is continuous for $y>1$.

Proceeding as with (D7), and assuming $y<1$, we find $\tilde{F}_{+}(y)=\tilde{F}_{+1}(y)+\tilde{F}_{+2}(y)$, where we omit the lengthy expression for $\tilde{F}_{+1}(y)$ but just note that it satisfies $\tilde{F}_{+1}(y) \rightarrow 0$ as $y \rightarrow 0$, and

$$
\tilde{F}_{+2}(y)=\frac{4}{g^{2}(y) B^{2}(y)}\left[\int_{0}^{y} d z B^{\prime}(z) J(z)-B(y) J(y)\right],
$$

where

$$
J(y)=\int_{0}^{y} d t \cos t B^{\prime}(y-t) \frac{g(y)-g(y-t)}{t} .
$$

No assumptions about the sign of $g^{\prime \prime \prime}(y)$ have been made yet. We now assume that $g^{\prime \prime \prime}(y) \geq 0$ for sufficiently small positive $y$, and we take $y$ to be so small that (D10) hold, $\cos y \geq 1 / 2$, and $g^{\prime} \leq 1 / 2$, the last of which implies $B^{\prime \prime}>0$. Differentiating (D18) and using (D10), we then have $J^{\prime}(y) \geq \frac{1}{2} B(y) / y$. Using (D17), and noting that the square brackets therein have the derivative $-B(y) J^{\prime}(y)$, L'Hôpital hence shows that $g(y) \tilde{F}_{+2}(y) \rightarrow-\infty$ as $y \rightarrow 0$.

Collecting, these observations show that $\tilde{F}(y)$ is continuous for $y>0$, but $\tilde{F}(y) \rightarrow-\infty$ as $y \rightarrow 0$, faster than any negative multiple of $1 / g(y)$.

\section{APPENDIX E: INTEGRALS}

In this appendix we collect results about integrals that appear in Sec. IV and Appendix C. We recall that $C_{K}$ (2.22) is smooth in $K$, it falls off at large $K$ faster than any inverse power of $K$, and $C_{0}=1$.

Proposition E.1 For $\alpha, \beta>0$, we have

$$
\begin{gathered}
\int_{0}^{\infty} \frac{d K}{K}\left(e^{i \alpha K}-e^{i \beta K}\right)=\ln (\beta / \alpha), \\
\int_{0}^{\infty} \frac{d K}{K}\left(e^{i \alpha K}-e^{-i \beta K}\right)=\ln (\beta / \alpha)+i \pi \\
\int_{0}^{\infty} \frac{d K}{K}\left[\left|C_{K}\right|^{2}-\cos (\alpha K)\right]=\ln \alpha+k_{1},
\end{gathered}
$$

where the integrals are improper Riemann integrals,

$$
k_{1}=\gamma+\int_{0}^{1} \frac{d K}{K}\left(\left|C_{K}\right|^{2}-1\right)+\int_{1}^{\infty} \frac{d K}{K}\left|C_{K}\right|^{2}
$$

and $\gamma$ is Euler's constant.

Proof.-In (E1a) and (E1b), we insert a low $K$ cutoff, express the integral of each term in terms of the exponential integral $E_{1}$ [36], and use small argument form of $E_{1}$ to remove the cutoff.

In (E1c), we break the integral into the subintervals $0<K<1$ and $1<K<\infty$, express the contributions from the subintervals in terms of the cosine integrals $\mathrm{Cin}$ and $\mathrm{Ci}$ [36], and use the cosine integral identities [36]. Note that $k_{1}$ is finite because of the small and large $K$ properties of $C_{K}$.

Proposition E.2. For $\alpha>0$, let

$$
H(\alpha):=\int_{0}^{\infty} \frac{d K}{K}\left(1-C_{K}\right) e^{i \alpha K}
$$

where the integral is an improper Riemann integral. Then 


$$
H(\alpha)= \begin{cases}-\int_{0}^{1} d z \frac{B(\alpha)-B(z)}{\alpha-z}+(1-B(\alpha))\left(\ln \left(\alpha^{-1}-1\right)+i \pi\right) & \text { for } 0<\alpha<1 ; \\ -\int_{0}^{1} d z \frac{B(\alpha)-B(z)}{\alpha-z} & \text { for } \alpha \geq 1 .\end{cases}
$$

It follows that $H$ is $C^{\infty}, H(\alpha)$ is real for $\alpha \geq 1$, and $H(\alpha)$ for $\alpha>1$ has the absolutely convergent series representation

$$
H(\alpha)=-\sum_{p=0}^{\infty} \frac{1}{\alpha^{p+1}} \int_{0}^{1} d z z^{p}(1-B(z)) .
$$

Proof.-Consider first $\operatorname{Im} H(\alpha)$. Taking the imaginary part of (E3) under the integral, recalling that $\int_{0}^{\infty} d K \sin (\alpha K) / K=$ $\pi / 2$ (since $\alpha>0$ by assumption), and introducing a large $K$ cutoff $M>0$, we have

$$
\operatorname{Im} H(\alpha)=\frac{\pi}{2}+\lim _{M \rightarrow \infty} I(M, \alpha),
$$

where

$$
\begin{aligned}
I(M, \alpha) & :=-\int_{0}^{M} \frac{d K}{K} \int_{0}^{1} d z B^{\prime}(z) \sin ((\alpha-z) K)=-\int_{0}^{1} d z B^{\prime}(z) \int_{0}^{M} \frac{d K}{K} \sin ((\alpha-z) K)=-\int_{0}^{1} d z B^{\prime}(z) \operatorname{Si}((\alpha-z) M) \\
& =-\operatorname{Si}((\alpha-1) M)-\int_{0}^{1} d z B(z) \frac{\sin ((\alpha-z) M)}{\alpha-z} \\
& =-\operatorname{Si}((\alpha-1) M)-B(\alpha) \int_{0}^{1} d z \frac{\sin ((\alpha-z) M)}{\alpha-z}+\int_{0}^{1} d z \frac{B(\alpha)-B(z)}{\alpha-z} \sin ((\alpha-z) M) \\
& =(B(\alpha)-1) \operatorname{Si}((\alpha-1) M)-B(\alpha) \operatorname{Si}(\alpha M)+\int_{0}^{1} d z \frac{B(\alpha)-B(z)}{\alpha-z} \sin ((\alpha-z) M)
\end{aligned}
$$

The first equality in (E7) is a definition, the second equality comes by interchanging the integrals, justified by the absolute convergence of the double integral, and the third equality uses the definition of the sine integral function $\mathrm{Si}$ [36]. The fourth equality comes from integration by parts, the fifth equality by decomposing the integrand, and the sixth equality by using again the definition of $\mathrm{Si}$. In the last expression in (E7), the integral term vanishes as $M \rightarrow$ $\infty$ by the Riemann-Lebesgue lemma, and since $\operatorname{Si}(x) \rightarrow$ $\pm \pi / 2$ as $x \rightarrow \pm \infty$ [36], the other two terms show that $I(M, \alpha) \rightarrow-\pi B(\alpha)+\pi / 2$ as $M \rightarrow \infty$. From this and (E6) we obtain the imaginary part of (E4).
Consider then $\operatorname{Re} H(\alpha)$. Taking the real part of (E2) under the integral, we introduce both a large $K$ cutoff and a small $K$ cutoff and proceed as above, using now the cosine integrals Cin and $\mathrm{Ci}$ [36]. Removing the cutoffs with the help of the cosine integral identities [36] gives the real part of (E4).

The smoothness of $H$ and the reality of $H(\alpha)$ for $\alpha \geq 1$ are immediate from (E4). The series (E5) follows from (E4) by writing $(\alpha-z)^{-1}=\alpha^{-1}(1-(z / \alpha))^{-1}$ and using the geometric series.
[1] L. Parker, Quantized fields and particle creation in expanding universes. 1., Phys. Rev. 183, 1057 (1969).

[2] G. Moore, Quantum theory of electromagnetic field in a variable-length one-dimensional cavity, J. Math. Phys. (N.Y.) 11, 2679 (1970).

[3] P. Candelas and D. Deutsch, On the vacuum stress induced by uniform acceleration or supporting the ether, Proc. R. Soc. A 354, 79 (1977).

[4] C. M. Wilson, G. Johansson, A. Pourkabirian, M. Simoen, J. R. Johansson, T. Duty, F. Nori, and P. Delsing,
Observation of the dynamical Casimir effect in a superconducting circuit, Nature (London) 479, 376 (2011).

[5] S. W. Hawking, Particle creation by black holes, Commun. Math. Phys. 43, 199 (1975); Erratum, Commun. Math. Phys. 46, 206(E) (1976).

[6] F. Belgiorno, S. L. Cacciatori, M. Clerici, V. Gorini, G. Ortenzi, L. Rizzi, E. Rubino, V. G. Sala, and D. Faccio, Hawking Radiation from Ultrashort Laser Pulse Filaments, Phys. Rev. Lett. 105, 203901 (2010). 
[7] J. Steinhauer, Observation of thermal Hawking radiation and its entanglement in an analogue black hole, Nat. Phys. 12, 959 (2016).

[8] S. L. Braunstein, Black hole entropy as entropy of entanglement, or it's curtains for the equivalence principle, arXiv:0907.1190v1; S. L. Braunstein, S. Pirandola, and K. Zyczkowski, Better Late than Never: Information Retrieval from Black Holes, Phys. Rev. Lett. 110, 101301 (2013).

[9] S.D. Mathur, The information paradox: a pedagogical introduction, Classical Quantum Gravity 26, 224001 (2009).

[10] A. Almheiri, D. Marolf, J. Polchinski, and J. Sully, Black holes: Complementarity or firewalls?, J. High Energy Phys. 02 (2013) 062.

[11] L. Susskind, Black hole complementarity and the HarlowHayden conjecture, arXiv:1301.4505.

[12] A. Almheiri, D. Marolf, J. Polchinski, D. Stanford, and J. Sully, An apologia for firewalls, J. High Energy Phys. 09 (2013) 018.

[13] J. Hutchinson and D. Stojkovic, Icezones instead of firewalls: Extended entanglement beyond the event horizon and unitary evaporation of a black hole, Classical Quantum Gravity 33, 135006 (2016).

[14] D. Harlow, Jerusalem lectures on black holes and quantum information, Rev. Mod. Phys. 88, 015002 (2016).

[15] N. D. Birrell and P.C. W. Davies, Quantum Fields in Curved Space (Cambridge University Press, Cambridge, England, 1982).

[16] R. M. Wald, Quantum Field Theory in Curved Spacetime and Black Hole Thermodynamics (University of Chicago Press, Chicago, 1994).

[17] L. Parker and D. Toms, Quantum Field Theory in Curved Spacetime (Cambridge University Press, Cambridge, England, 2009).

[18] J. Louko, Unruh-DeWitt detector response across a Rindler firewall is finite, J. High Energy Phys. 09 (2014) 142.

[19] E. Martín-Martínez and J. Louko, $(1+1)$ D Calculation Provides Evidence that Quantum Entanglement Survives a Firewall, Phys. Rev. Lett. 115, 031301 (2015).

[20] W. G. Unruh, Firewalls-A gravitational perspective, lecture at RQIN-2014 (Seoul, Korea, July 2014).

[21] E. G. Brown, M. del Rey, H. Westman, J. León, and A. Dragan, What does it mean for half of an empty cavity to be full?, Phys. Rev. D 91, 016005 (2015).
[22] E. G. Brown and J. Louko, Smooth and sharp creation of a Dirichlet wall in $1+1$ quantum field theory: how singular is the sharp creation limit?, J. High Energy Phys. 08 (2015) 061.

[23] T. Harada, S. Kinoshita, and U. Miyamoto, Vacuum excitation by sudden appearance and disappearance of a Dirichlet wall in a cavity, Phys. Rev. D 94, 025006 (2016).

[24] W. G. Unruh, Notes on black hole evaporation, Phys. Rev. D 14, 870 (1976).

[25] B. S. DeWitt, in General Relativity: An Einstein Centenary Survey, edited by S. W. Hawking and W. Israel (Cambridge University Press, Cambridge, England, 1979).

[26] R. Wong, Asymptotic Approximations of Integrals (Society for Industrial and Applied Mathematics, Philadelphia, 2001).

[27] A. Satz, Then again, how often does the Unruh-DeWitt detector click if we switch it carefully?, Classical Quantum Gravity 24, 1719 (2007).

[28] J. Louko and A. Satz, Transition rate of the Unruh-DeWitt detector in curved spacetime, Classical Quantum Gravity 25, 055012 (2008).

[29] R. H. Jonsson, E. Martín-Martínez, and A. Kempf, Information Transmission without Energy Exchange, Phys. Rev. Lett. 114, 110505 (2015).

[30] A. Blasco, L. J. Garay, M. Martín-Benito, and E. MartínMartínez, Violation of the Strong Huygen's Principle and Timelike Signals from the Early Universe, Phys. Rev. Lett. 114, 141103 (2015).

[31] A. Blasco, L. J. Garay, M. Martín-Benito, and E. MartínMartínez, Timelike information broadcasting in cosmology, Phys. Rev. D 93, 024055 (2016).

[32] M. Reed and B. Simon, Methods of Modern Mathematical Physics II: Fourier Analysis, Self-adjointness (Academic, New York, 1975).

[33] J. Blank, P. Exner, and M. Havlíček, Hilbert Space Operators in Quantum Physics, 2nd edition (Springer, New York, 2008).

[34] G. Bonneau, J. Faraut, and G. Valent, Self-adjoint extensions of operators and the teaching of quantum mechanics, Am. J. Phys. 69, 322 (2001).

[35] G. Kunstatter, J. Louko, and J. Ziprick, Polymer quantization, singularity resolution and the $1 / r^{2}$ potential, Phys. Rev. A 79, 032104 (2009).

[36] NIST Digital Library of Mathematical Functions. http:// dlmf.nist.gov/, Release 1.0.13 of 2016-09-16. 